\title{
Median canaliform dystrophy of Heller
}

\section{Juliandri Juliandri, Yang Xu}

Department of Dermatology, The First Affiliated Hospital of Nanjing Medical University, Nanjing, Jiangsu Province, China

Corresponding author: Yang Xu, MD, E-mail: cardioos@hotmail.com

\begin{abstract}
Median Canaliform Dystrophy of Heller is characterized by midline longitudinal furrow with multiple transverse parallel lines. Repetitive trauma to the nail plate and cuticle may be responsible for some cases, however some case reports have suggested familial occurrence and use of oral retinoids in its causation. We present a special case of Median Canaliform Dystrophy of Heller in middle age Chinese male and discuss the possibility cause in our case. Median Canaliform Dystrophy of Heller case occurred in Chinese patient that has no special related diseases and it resolved simultaneously and happened again.
\end{abstract}

Key words: Median nail dystrophy; Nail plate; Cuticle

\section{INTRODUCTION}

Median Canaliform Dystropy of Heller or Onychodystrophia Mediana Canaliformis is a relatively rare albeit striking habit tic deformity of thumb nails characterized by midline longitudinal furrow with multiple transverse parallel lines. Although the proposed etiopathogenesis is repetitive trauma in the form of pushing down the cuticle and proximal nail fold, in majority of cases the cause may be obscure. Some case reports have suggested familial occurrence and use of oral retinoids in its causation [1]. Histopathology classically shows parakeratosis, accumulation of melanin within and between the nail bed keratinocytes [2]. Treatment is often prolonged and unsatisfactory, though topical agents like $0.1 \%$ tacrolimus have been used successfully [1]. Psychiatric opinion should be taken when associated with the depressive, obsessive-compulsive, or impulse-control disorder. We report a case of 35-year-old Chinese male diagnosed as median nail dystrophy.

\section{CASE REPORT}

A 35 year old Chinese male is presented to Dermatology Outpatient Department with complaints of lesion over his thumb nail since 6 months. The patient denied history of intentional pushing down of cuticular portion of proximal nail fold. The patient had such condition about one year ago and it resolved simultaneously within several months, and had it again. No history of taking oral retinoids or other medications, or history of contact with irritatnts or allergens was present. The patient didn't have any family history of nail disorders. On examination, there was median split of right thumb nail with transverse furrows extending from longitudinal split (Fig. 1). Rest other finger and toe nails were normal. Systemic examination was unremarkable. The patient refused biopsy. Diagnosis of median nail dystrophy was made on a clinical basis. The patient is still in follow up.

Discussion Median canaliform dystrophy of Heller, also known as dystrophia unguis mediana canaliformis, solenonychina, and nevus striatus unguis, is a dystrophic condition of the nail in which longitudinal splitting occurs [3]. It is almost exclusively seen on the thumbs, often bilaterally. It is characterized by an inverted fir tree-like split or canaliform in the nail plate [3]. The exact etiology of this intriguing condition is yet to be elucidated. However, subungual skin tumors such as glomus tumors, myxoid tumors, have also been described to cause longitudinal grooving, lifting of the nail plate from the bed resulting in a tube-like

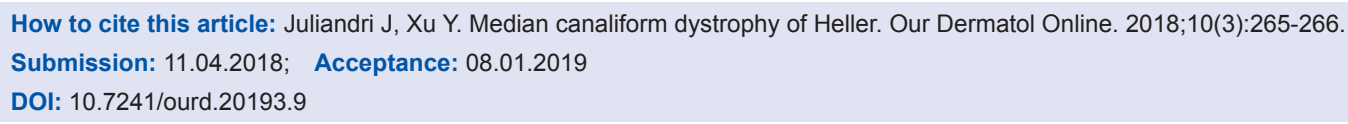




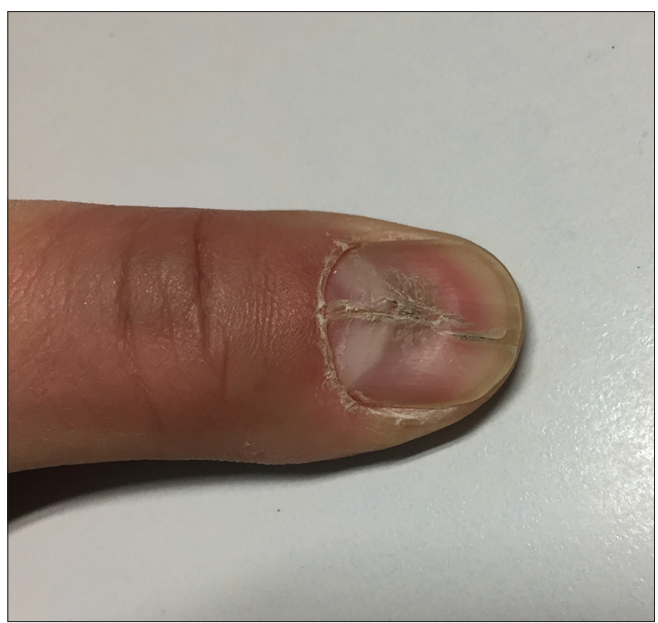

Figure 1: Median split of right thumb nail with transverse furrows extending from longitudinal split.

structure (solenos) distal to it [3]. To our knowledge, only 6 cases have been reported in literature. 4 reported cases occurred in adults and 2 reported case occurred in children. 5 reported cases are associated with trauma. Wu et al (2009) reported a 56 year old female presented with median canaliform dystrophy of Heller with associated swan neck deformity. She had a history of repeated minor injuries over both hands. She didn't have a history of taking any oral medication or have contact with any irritants and no familial history [3]. Madke et al reported a 25 year old female presented with backwardly-angled ridges on her thumbnails resembling a fir tree. She denied a history of repetitive trauma. The patient didn't report any history of contact with known allergens and irritants. She didn't have any family history of nail disorder. The patient was then prescribed topical tazarotene $0.05 \%$ ointment to be applied at bedtime and was asked to follow-up [4]. Avhad et al reported a 5 year old Indian boy was presented with single dystrophic thumb nail. He had history of constant biting of thumb nails and was also diagnosed to have attention deficit hyperactive disorder (ADHD) [5]. Kola et al reported a 19 year old female presented with median nail dystrophy. History of biting of thumb nails was present. No history of use of oral retinoids or other medications, or history of contact with irritants or allergens was present. He denied a family history. The patient then was prescribed with $0.1 \%$ tacrolimus ointment topically at night and showed some improvement. The patient is still in follow up.[2] Pathania et al reported a 22 year old
Indian male was diagnosed to have Median Canaliform Dystrophy of Heller that affecting both great toe nails and great thumb nails. He was then prescribed topical $0.1 \%$ Tacrolimus ointment and Oral Fluoxetine at the dose of $50 \mathrm{mg}$ daily. However, the case was lost to follow up after putting the patient first review [1]. Damevska et al reported 11 year old girl who developed canaliform dystrophy and long-term hypopigmentation following cryotheraphy of warts on proximal nail folds [6]. In our patient described herein, this case is special because self-inflicting nail trauma involving manipulation of cuticular portion of proximal nail fold and familial were not present. As the medication history was also absent, and it could resolve simultaneously, also tumor is not considered. The possibility cause in this case remains idiopathic. The patient is still in follow-up.

\section{CONCLUSION}

In closing, we report a Median Canaliform Dystrophy of Heller case occurred in Chinese patient that has no special related diseases and it resolved simultaneously and happened again.

\section{Consent}

The examination of the patient was conducted according to the Declaration of Helsinki principles.

\section{REFERENCES}

1. Pathania V. Median Canaliform Dystrophy of Heller occurring on thumb and great toe nails. Med J Armed Forces India. 2016;72:178-9.

2. Kota R, Pilani A, Nair PA. Median Nail Dystrophy Involving the Thumb Nail. Indian J Dermatol. 2016;61:120.

3. Wu CY, Chen GS, Lin HL. Median canaliform dystrophy of Heller with associated swan neck deformity. J Eur Acad Dermatol Venereol. 2009;23:1102-3.

4. Madke B, Gadkari R, Nayak C. Median canaliform dystrophy of Heller. Indian Dermatol Online J. 2012;3:224-5.

5. Avhad G, Ghuge P. Median canaliform dystrophy of Heller. Indian Pediatr. 2013;50:1073.

6. Damevska K, Duma S, Pollozhani N. Median canaliform dystrophy of Heller after cryotherapy. Pediatr Dermatol. 2017;34:726-7.

Copyright by Juliandri Juliandri, et al. This is an open-access article distributed under the terms of the Creative Commons Attribution License, which permits unrestricted use, distribution, and reproduction in any medium, provided the original author and source are credited.

Source of Support: Nil, Conflict of Interest: None declared. 